\title{
Single-molecule dynamics of nuclear mRNA
} Yaron Shav-Tal ${ }^{1 *}$ and Yosef Gruenbaum ${ }^{2}$

\author{
Addresses: ${ }^{1}$ The Mina and Everard Goodman Faculty of Life Sciences and Institute of Nanotechnology, Bar-Ilan University, Ramat Gan 52900 , \\ Israel; ${ }^{2}$ Department of Genetics, The Institute of Life Sciences, The Hebrew University of Jerusalem, Givat Ram, Jerusalem 91904, Israel \\ *Corresponding author: Yaron Shav-Tal (shavtaly@mail.biu.ac.il) \\ FI000 Biology Reports 2009, I:29 (doi:I0.34I0/BI-29)
}

The electronic version of this article is the complete one and can be found at: http://FI000.com/Reports/Biology/content/I/29

\begin{abstract}
In eukaryotes, mRNA molecules are transcribed from nuclear DNA and commute through a labyrinth of nucleoplasmic passageways to the nuclear envelope where they are exported to the cytoplasm. New findings provide tools and insights into the biophysical properties that govern mRNA translocations en route to the cytoplasm and suggest that mRNA molecules move in a discontinuous manner due to transient interactions with the nuclear environment.
\end{abstract}

\section{Introduction and context}

During interphase, RNA polymerase II transcribes numerous gene loci and generates thousands of mRNA molecules that are exported to the cytoplasm. Transcription occurs in conjunction with capping, splicing, polyadenylation and packaging with ribonuclear proteins [heterogeneous nuclear ribonucleoproteins (hnRNPs)] [1,2]. Correct packaging of the released mRNA molecules, termed messenger ribonulcear particles (mRNPs), probably yields export-competent complexes that can efficiently interact and pass through the nuclear pore complex (NPC) into the cytoplasm [3].

Issues such as mRNP accessibility, nuclear constraints, nucleoplasmic viscosity, and mobility kinetics must be pursued in order to understand the biophysical rules that govern the journey of mRNPs. Putting it simply, a voyage of many hurdles can be envisaged, considering the nuclear environment in which mRNPs must travel, which harbors dense chromatin regions, nucleoli, nuclear bodies, nuclear scaffolds and other constituents of inter-chromatin space [4]. Moreover, the intranuclear mobility of the aforementioned bodies and domains adds another level of complexity to our understanding of nuclear trafficking [5]. The presence or lack of either a 'guiding hand' or a functional network of filaments assisting nuclear mRNPs in reaching their cytoplasmic destiny is of fundamental interest [6].
Nuclear tracks between chromatin domains, in which mRNAs could travel, were observed in fixed cells using fluorescent in situ hybridization (FISH) techniques [7-9]. Some years later, with the rise of RNA labeling techniques that allow detection within a living cell environment, the movement kinetics of mRNAs were analyzed directly. RNA FISH modified for tagging of mRNA in vivo using a fluorescent oligo(dT) probe was utilized in the first real-time measurements of poly(A) mRNA mobility [7]. Labeled RNA signals spread throughout the nucleus, suggesting random motion, typically referred to as diffusion, which indicates that the movement is due to thermal energy within the environment. Analysis by fluorescence correlation spectroscopy (FCS) yielded average diffusion coefficients of $0.6 \pm 0.1$ to $0.7 \pm 0.19 \mathrm{\mu m}^{2} / \mathrm{s}$ for polyadenylated mRNAs within the nucleoplasm $[8,9]$. Interestingly, there was no difference in mRNA mobility as it moved in and out of nuclear bodies known as nuclear speckles [9-11]. Using a photobleaching approach [fluorescence recovery after photobleaching (FRAP)], the diffusion properties of polyadenylated mRNAs tagged with a green fluorescent protein (GFP)-fusion of the poly(A)-binding protein yielded diffusion coefficients of approximately $0.04 \mathrm{\mu m}^{2} / \mathrm{s}[12,13]$. FRAP of in vivo FISH-labeled poly (A) mRNAs also measured an average diffusion coefficient of $0.03 \mu \mathrm{m}^{2} / \mathrm{s}$ [11]. The disparity in measurements might be explained by the different sensitivities of the 
methods used (see discussions in [11] and [9]). These studies confirmed the notion that mRNA mobility in the nucleus follows a random path throughout interchromatin space [8], yet did not concur whether this was an energy-dependent process.

By introducing repeats of a bacteriophage RNA binding sequence (MS2) into a particular gene in human cells, single mRNA molecules transcribed from a particular gene could be tagged with many GFP-labeled MS2 binding proteins, thereby yielding a system in which single nuclear mRNPs could be discerned and tracked [14]. Single-particle tracking (SPT) and mean square displacement analysis (MSD) for modeling of the mobility showed that mRNPs move by simple diffusion (Figure 1). Some of the movements were spatially restricted due to the presence of the chromatin barriers bordering the inter-chromatin space. Diffusion coefficients measured by SPT averaged $0.04 \mu^{2} / \mathrm{s}$, and FRAP measurements of the whole population of these transcripts indicated that faster movements also exist. In another study, molecular beacons were used to tag 96 sequence repeats within a particular mRNA species. SPT and MSD analysis showed that they diffuse at an average rate of $0.03 \mu \mathrm{m}^{2} / \mathrm{s}$ [15]. Both studies demonstrated that mRNP movement in the nucleus does not require energy. Furthermore, directional mRNP movements were not identified, arguing against the presence of a filamentous network for nuclear mRNA trafficking. Still, it is possible that the tracking methods applied were not capable of capturing very rapid mRNA movements and, therefore,

Figure I. Mean square displacement analysis
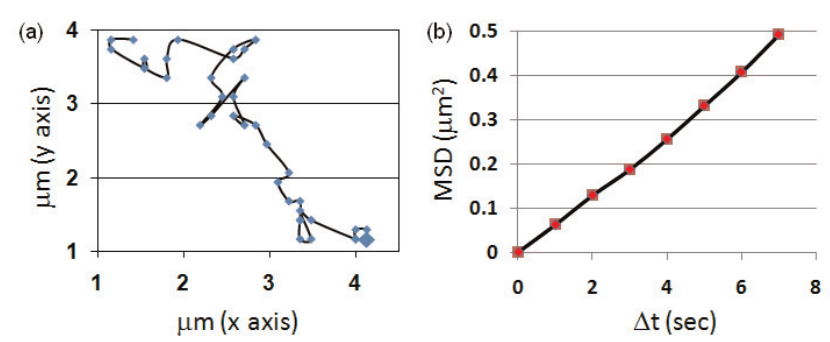

A single particle (for example, an mRNP) is tracked (SPT) in two dimensions $(x, y)$ in a series of images from a two-dimensional time lapse movie. The $x, y$ pixel values are recorded and (a) the distance of movement is plotted. The time interval between each image is denoted as $\Delta t$. The change in the distance (displacement) of the particle between each image is measured. This is repeated for each image and an MSD value is obtained. Then, the same displacement measurements are performed when the $\Delta t$ value equals $2 \Delta \mathrm{t}, 3 \Delta \mathrm{t}$ and so forth. (b) The MSD value that was calculated for each $\Delta \mathrm{t}$ interval is plotted. A linear relationship between the MSD and $\Delta t$ means that random movement has been tracked. MSD, mean square displacement; SPT, single particle tracking; $t$, time. could provide reliable results only for the population of moderate to slow moving mRNAs.

\section{Major recent advances}

The above approaches complement each other, yet could not provide information on the specific dynamics of single molecules of an endogenous mRNA species. Recently, such data have finally been obtained. The model system used comprised the unique nuclei present in the salivary glands of the dipteran Chironomus tentans. The large nucleoplasm contains four distinct giant polytene chromosomes, which transcribe large mRNAs called Balbiani ring (BR) mRNAs that encode secretory proteins. BR mRNAs (30-40 kb) are mostly exonic, and are assembled into large mRNPs $(50 \mathrm{~nm})$. Elegant electron microscope work has shown how BR granules are formed during transcription, move in the nucleus, and traverse the NPCs $[16,17]$. BR mRNP movement in the nucleoplasm was assessed using fixed electron microscopy specimens. Comparison of mRNP dispersal in the nucleus at different time-points after start of transcription showed a random pattern of movement (estimated $D=0.12 \mu \mathrm{m}^{2} / \mathrm{s}$ ) [18].

Now Kubitscheck, Daneholt and colleagues [19] have taken this analysis one step further using a highresolution live-cell imaging approach. In order to label the endogenous BR transcripts, they used two different techniques: an in vivo RNA FISH approach in which they utilized the binding of a fluorescent probe (DNA or RNA) to a repetitive sequence in the BR2 mRNA; and a tagged-protein approach in which they injected a fluorescent hnRNP A1-like protein, hrp36, which binds repetitively to BR2 mRNA [20]. Two microscope systems were used for imaging, a high-speed laser scanning microscope and an ultrasensitive single-molecule microscope [21]. In order to track single mRNP movements, the authors had to overcome the vast amount of signal emanating from many BR mRNPs. This was achieved by photobleaching a portion of the nucleoplasm and subsequently imaging fluorescent single mRNPs that translocated into the photobleached zone. mRNPs were detected moving throughout the whole nucleoplasm and interacting with NPCs, but were excluded from the chromosome areas. In order to obtain reliable data on mRNP translocations, the authors not only used MSD analysis but put emphasis on measuring the total distribution of the actual mRNP 'jumping distance' between each acquired frame. The jump-distance approach enables a closer look at a heterogeneous population of molecules or movements, and was previously used by the authors to obtain high-resolution single-molecule measurements of the nucleoplasmic microenvironment in mammalian cells [22]. 
The authors present some interesting conclusions. The C. tentans nucleoplasm is devoid of chromatin but has a viscosity (3-5 cP) similar to that of mammals $(4-8 \mathrm{cP})$. Within the nucleoplasmic space four types of diffusional mobility were detected: a notably fast fraction $(D=2.7$ $\left.4.7 \mathrm{\mu m}^{2} / \mathrm{s}\right)$, and three slower ones $(D=0.6,0.02$, and $0.015 \mathrm{\mu m}^{2} / \mathrm{s}$ ). Using simulations, they show that these reflect different portions of an mRNP track, rather than different types of mRNPs. This in turn implies that mRNP motion is abrogated either due to physical constraints in the microenvironment or through transient associations of mRNPs with a nuclear structure. The latter explanation is favored since BR mRNPs that are associated with a non-chromatin fibrous network have been detected [23]. Still, the situation in C. tentans differs somewhat from the mammalian nucleus, in which considerably slower diffusion coefficients have been measured. These most probably reflect the crowded chromatin environment through which the mRNPs must navigate until they reach the NPC.

\section{Future directions}

Nuclear mRNA moves by diffusion, but do other types of motion exist, namely directional movement? Transcription-associated myosin motors and actin monomers are present in the nucleus, and it is thus enticing to assume that some cross-talk between mRNA mobility and motor-driven trafficking exists. Enhanced imaging resolution and acquisition speeds for single molecule analysis will enable further examination of the trafficking kinetics of different endogenous transcripts within their natural microenvironments, as well as a better comprehension of the inter-chromatin media through which they travel.

\section{Abbreviations}

$\mathrm{BR}$, Balbiani ring; FCS, fluorescence correlation spectroscopy; FISH, fluorescence in situ hybridization; FRAP, fluorescence recovery after photobleaching; GFP, green fluorescent protein; hnRNP, heterogeneous nuclear ribonucleoprotein; mRNP, messenger ribonulcear particle; MSD, mean square displacement; NPC, nuclear pore complex; SPT, single particle tracking.

\section{Competing interests}

The authors declare that they have no competing interests.

\section{Acknowledgements}

YS-T is supported by the Israel Science Foundation (ISF) (250/06), ISF-Bikura, Israel Cancer Research Fund (ICRF), German-Israeli Foundation for Scientific Research and Development (GIF), USA-Israel Binational
Science Foundation (BSF), German-Israeli Project Cooperation (DIP), and the Israeli Ministries of Science and Health, and is the Jane Stern Lebell Family Fellow in Life Sciences at Bar-Ilan University. YG is supported by the Muscular Dystrophy Association (MDA4329), GIF, BSF, ISF, ISF-Morasha, the EU's Sixth Framework Programme (FP6) (EURO-Laminopathies), the Fritz-Theissen Foundation, and by the Israeli Ministry of Health.

\section{References}

I. Neugebauer KM: On the importance of being co-transcriptional. J Cell Sci 2002, I I5:3865-7I.

2. Dreyfuss G, Kim VN, Kataoka N: Messenger-RNA-binding proteins and the messages they carry. Nat Rev Mol Cell Biol 2002, 3:195-205.

3. Moore MJ: From birth to death: the complex lives of eukaryotic mRNAs. Science 2005, 309: |5|4-8.

4. Cremer T, Cremer C: Chromosome territories, nuclear architecture and gene regulation in mammalian cells. Nat Rev Genet 2001, 2:292-301.

5. Shav-Tal $Y$, Darzacq $X$, Singer $\mathrm{RH}$ : Gene expression within a dynamic nuclear landscape. EMBO J 2006, 25:3469-79.

6. Pederson T: Half a century of "the nuclear matrix". Mol Biol Cell 2000, I I:799-805.

7. Politz JC, Browne ES, Wolf DE, Pederson T: Intranuclear diffusion and hybridization state of oligonucleotides measured by fluorescence correlation spectroscopy in living cells. Proc Natl Acad Sci U S A 1998, 95:6043-8.

8. Politz JC, Tuft RA, Pederson T, Singer RH: Movement of nuclear poly(A) RNA throughout the interchromatin space in living cells. Curr Biol 1999, 9:285-91.

9. Politz JC, Tuft RA, Prasanth KV, Baudendistel N, Fogarty KE, Lifshitz LM, Langowski J, Spector DL, Pederson T: Rapid, diffusional shuttling of poly(A) RNA between nuclear speckles and the nucleoplasm. Mol Biol Cell 2006, I 7:1239-49.

10. Lamond Al, Spector DL: Nuclear speckles: a model for nuclear organelles. Nat Rev Mol Cell Biol 2003, 4:605-I2.

II. Molenaar C, Abdulle A, Gena A, Tanke HJ, Dirks RW: Poly(A)+ RNAs roam the cell nucleus and pass through speckle domains in transcriptionally active and inactive cells. J Cell Biol 2004, 165:191-202.

FI000 Factor 6.0 Must Read

Evaluated by Thomas Cremer 07 Jul 2004

12. Calapez A, Pereira HM, Calado A, Braga J, Rino J, Carvalho C, Tavanez JP, Wahle E, Rosa AC, Carmo-Fonseca M: The intranuclear mobility of messenger RNA binding proteins is ATP dependent and temperature sensitive. J Cell Biol 2002, I 59:795-805.

13. Braga J, McNally JG, Carmo-Fonseca M: A reaction-diffusion model to study RNA motion by quantitative fluorescence recovery after photobleaching. Biophys J 2007, 92:2694-703.

14. Shav-Tal Y, Darzacq X, Shenoy SM, Fusco D, Janicki SM, Spector DL, Singer RH: Dynamics of single mRNPs in nuclei of living cells. Science 2004, 304: 1797-800.

15. Vargas DY, Raj A, Marras SA, Kramer FR, Tyagi S: Mechanism of mRNA transport in the nucleus. Proc Natl Acad Sci U S A 2005, 102: I7008-I3

16. Daneholt B: Assembly and transport of a premessenger RNP particle. Proc Natl Acad Sci U S A 200I, 98:70I2-7.

17. Mehlin H, Daneholt B, Skoglund U: Translocation of a specific premessenger ribonucleoprotein particle through the nuclear pore studied with electron microscope tomography. Cell 1992, 69:605-I3. 
18. Singh OP, Bjorkroth B, Masich S, Wieslander L, Daneholt B: The intranuclear movement of Balbiani ring premessenger ribonucleoprotein particles. Exp Cell Res 1999, 25 I:135-46.

19. Siebrasse JP, Veith R, Dobay A, Leonhardt H, Daneholt B, Kubitscheck U: Discontinuous movement of mRNP particles in nucleoplasmic regions devoid of chromatin. Proc Natl Acad Sci U S A 2008, 105:20291-6.

20. Visa N, Alzhanova-Ericsson AT, Sun X, Kiseleva E, Bjorkroth B, Wurtz T, Daneholt B: A pre-mRNA-binding protein accompanies the RNA from the gene through the nuclear pores and into polysomes. Cell 1996, 84:253-64.
2I. Siebrasse JP, Grunwald D, Kubitscheck U: Single-molecule tracking in eukaryotic cell nuclei. Anal Bioanal Chem 2007, 387:41-4.

22. Grunwald D, Martin RM, Buschmann V, Bazett-Jones DP, Leonhardt H, Kubitscheck U, Cardoso MC: Probing intranuclear environments at the single-molecule level. Biophys J 2008, 94:2847-58.

23. Miralles F, Ofverstedt LG, Sabri N, Aissouni Y, Hellman U, Skoglund $\mathrm{U}$, Visa N: Electron tomography reveals posttranscriptional binding of pre-mRNPs to specific fibers in the nucleoplasm. J Cell Biol 2000, I48:27I-82. 